\section{Social facilitation of the rat's responding in extinction}

\author{
F. ROBERT TREICHLER, MARILYN M. GRAHAM \\ and \\ G. EDWARD SCHWEIKERT III \\ Kent State University, Kent, Ohio 44240
}

The extinction performances of rats were compared in several experiments which provided either individual or paired conditions during operant acquisition and/or extinction. Enhanced resistance to extinction was observed when Ss were trained alone and, subsequently, extinguished in pairs. All other combinations of conditions yielded lower and nondifferential levels of responding during testing. This pattern of results did not appear specific to condition-dependent changes in the nature of stimuli associated with reinforcement. It was considered that the results could be attributed to a limited-term arousal effect of social pairing.

Zajonc (1965) has reviewed a variety of experiments on social influences on performance and has suggested that at least one function of the presence of a second S may be to provide arousal consequences. Further, Zajonc \& Sales (1966) have considered that this arousal is analogous to the "generalized drive" of classical motivation-learning theories and that, like $D$, it may enhance or impair performance in different task situations.

The present study attempted to assess such social influences on extinction, and the experiments were devised to provide what Hall (1962) has described as "factorial" investigations of motivational consequences. Two general questions about social effects on performance were posed. First, does the coactive presence of one rat operate as a response facilitator for another rat during the extinction of a simple operant? Second, is this influence independent of the kind of social conditions that prevailed during the acquisition of this response?

EXPERIMENT 1

Subjects

Seventy-two adult male Holtzman albino rats served as Ss.

Apparatus

All testing was done in a modified Scientific Prototype Skinner box. The usual bar was made inoperative, and the foodcup was replaced by a 3/4-in.-diam hollow Lucite rod which protruded $1 \mathrm{in.}$ through the wall. Discrete depressions of this rod were required to operate a food tispenser which delivered one Noyes rat-food pellet per press to a receptacle on the tip of the rod. All responses were registered on a Rustrak event recorder. Procedure

Pairs of Ss were matched by weight and caged together for at least 15 days prior to training. Each $\mathbf{S}$ was reduced to $90 \%$ of its predeprivation weight and was placed alone in the training and extinction conditions. Three squads of $24 \mathrm{Ss}$, with three pairs assigned to each condition, were concurrently tested. No indications of differences among the squads were observed, and all data were combined in analysis.

\section{Results}

While comparison of acquisition performances by Ss trained alone or together was not the primary concern of this experiment, only slight differences between these groups were noted during this phase of testing. The mean time required to complete training sessions was approximately twice as long for Ss trained together as for Ss trained alone, and this similarity of response rates was interpreted as an indication of little social influence upon acquisition. Further, unlike rhesus monkeys in similar situations (Horel, Treichler, \& Meyer, 1963), negligible competition for the manipulandum was observed between Ss undergoing concurrent acquisition. The general nature of responding in this phase seemed to be characterized by "timesharing" of the manipulandum, and we consider this property to be a consequence of providing the dispenser and manipulandum as a single unit in the apparatus.

Results from the extinction phase of this experiment are presented in Table 1A. This displays the mean numbers of responses and the amount of time required to meet the extinction criterion under the four treatments which result from the possible combinations of alone and together conditions in acquisition and extinction. Analyses of variance indicated significant differences with each dependent measure [responses, $F(3,63)=17.5$; time, $F(3,63)=19.8$, both ps $<.01]$, and Newman-Keuls comparisons showed that these differences were based solely upon the significantly $(p<.01)$ greater resistances to extinction observed when Ss were alone in acquisition and together in extinction. On neither of the measures did any other conditions vary from one another.

Because concurrent extinction in the "together" condition might have limited individual temporal availability of the response manipulandum, and, consequently, allowed pauses for dissipation of response-produced inhibition, the mean interresponse intervals under the various conditions were also compared. However, no significant differences were observed $[F(3,63)=1.81, \quad p<.05]$, and it appeared that rats emitted responses at similar rates whether alone or together in extinction. 
Table 1

Mean Number of Responses and Time (in Min) to Meet Extinction Criterion of No Responses in $3 \mathrm{Min}$ Under the Conditions of the Various Experiments. Significance Levels for Each Measure are Indicated in the Far Right Column.

\begin{tabular}{|c|c|c|c|c|c|c|}
\hline & $\begin{array}{l}\text { Acquisition Condition } \\
\text { Extinction Condition }\end{array}$ & $\begin{array}{l}\text { Alone } \\
\text { Alone }\end{array}$ & $\begin{array}{c}\text { Alone } \\
\text { Together }\end{array}$ & $\begin{array}{c}\text { Together } \\
\text { Alone }\end{array}$ & $\begin{array}{l}\text { Together } \\
\text { Together }\end{array}$ & $\mathrm{p}$ \\
\hline $\begin{array}{l}\text { A: Experiment } 1 \\
\text { Normal Click }\end{array}$ & Mean responses & 146 & 447 & 120 & 172 & $<.01$ \\
\hline $\mathrm{N}=18$ per group & Mean time & 18.6 & 50.4 & 14.9 & 17.2 & $<.01$ \\
\hline $\begin{array}{l}\text { B: Experiment } 2 \\
\text { Clicks Only }\end{array}$ & Mean responses & 135 & 124 & 138 & 104 & n.s. \\
\hline$N=12$ per group & Mean time & 16.3 & 15.8 & 12.6 & 13.9 & n.s. \\
\hline $\begin{array}{l}\text { C: Experiment } 3 \\
\text { No Clicks }\end{array}$ & Mean responses & 90 & 142 & 87 & 80 & $<.05$ \\
\hline$N=12$ per group & Mean time & 13.58 & 28.29 & 12.73 & 18.50 & $<.01$ \\
\hline
\end{tabular}

\section{EXPERIMENT 2}

A possible explanation of the results of Experiment 1 considers that some stimuli in the training situation might have provided influences that were unrelated to the imposed social conditions. Training alone always provided a secondary reinforcer, feeder click, with the delivery of food. Contrastingly, when animals were trained together, food was presented with the click on only about half of the click occurrences, i.e., when the other $\mathrm{S}$ responded, clicks, but not food, ensued.

When the Ss trained under these different conditions were subsequently extinguished, they may have responded to changes in the response-contingent nature of secondary reinforcement. For example, Ss trained alone and extinguished together may have reacted to the clicks produced by their partners as general arousal-eliciting stimuli, and this might have enhanced their responding in extinction. Since this group was, indeed, the one which displayed greatest resistance to extinction, a second experiment was designed to assess the influence of changes in reinforcement-related stimuli between acquisition and extinction sessions. This was accomplished by providing relationships between primary and secondary reinforcing stimuli that were identical to those of the various groups in Experiment 1, without providing the actual social conditions of that experiment.

Procedure

Forty-eight rats, divided into four groups of $12 \mathrm{Ss}$ each, were maintained and pretrained exactly as in Experiment 1 . The only differences in procedures were instituted when animals were trained or extinguished under conditions designed to be analogous to the "together" treatments of the previous experiment. Although animals were actually alone in the apparatus at all times, half of the Ss were presented with a sequence of nonreinforced feeder clicks during training. Temporal characteristics of this sequence were based on the interresponse intervals observed in Experiment 1, and, consequently, click occurrences were normally distributed about a mean of $6 \mathrm{sec}$, with a SD of $1.5 \mathrm{sec}$.

Half of the animals trained under this condition and half of those trained without the clock presentations ,were subsequently extinguished in the presence of nonresponse-contingent feeder click. Again, based upon the temporal response characteristics observed in Experiment 1, a sequence of click occurrences was generated. Since responses in extinction had been observed to be highly correlated within S pairs, this sequence involved a manual presentation of clicks which approximated the responding of the animal under extinction test. This was accomplished by first presenting clicks at the $S$ 's response rate and maintaining this until $S$ made two pauses of $5 \mathrm{sec}$ duration. Thereafter the number of responses was matched by clicks, with half presented when $\mathrm{S}$ was pausing and half when $S$ was responding, until a period of $2 \mathrm{~min}$ of no response was observed. Then a three-click burst was presented each minute until $S$ met the 3 -min no-response extinction criterion. All treatments involving alone conditions were exactly as in Experiment 1, and both times and number of responses to extinction were compared among the four groups derived from the possible combinations of training and extinction conditions.

\section{Results}

Table 1B displays the mean extinction scores from Experiment 2. Analyses of variance indicated no significant differences (both $F_{s}<1$; ps $>.05$ ) among the treatment groups on either measure.

\section{EXPERIMENT 3}

Since mere presentation of clicks without the presence of a partner did not appear to produce extinction differences like those of Experiment 1, Experiment 3 attempted to assess the independent influence of another animal's presence during extinction. Further, this test was designed to assess extinction performances when only minimal secondary reinforcing stimuli were provided.

\section{Procedure}

Forty-eight rats were again assigned to pairs, and conditions like those of the previous experiments were imposed. All pretraining and acquisition procedures were identical to those in Experiment 1. In extinction, however, the feeder was rendered inoperative in order to eliminate the secondary reinforcing stimuli of feeder clicks. Extinction measures like those of the previous experiments were recorded.

\section{Results}

Table $1 \mathrm{C}$ displays measures comparable to those of the two previous studies. Analyses of variance indicated significant differences among the scores obtained on both measures [responses, $\quad F(3,44)=3.38, \quad p<.05$; time, $F(3,44)=10.34, p<.01]$. For the number of responses to extinction measure, a subsequent Newman-Keuls comparison indicated the same pattern of intergroup differences as was present in Experiment 1 . On the time-to-extinction measure, the $\mathrm{Ss}$ trained alone and extinguished together were different from all other groups according to Newman-Keuls comparisons, and, additionally, the together-together group was different from both the groups extinguished alone.

In terms of our initial questions concerning social influences on performance in this task, it is concluded that enhanced resistance to extinction may be a consequence of the coactive presence of another animal. However, this result appears specific to extinction tests conducted after the $S$ has been alone during previous exposure to the training situation.

The results of Experiment 2 indicate that the differences in extinction measures are not dependent upon an artifactual influence produced by differential changes in the nature of secondary reinforcing stimuli when $\mathrm{Ss}$ are shifted from acquisition to extinction. Indeed, when secondary reinforcing stimuli are minimized, as in Experiment 3, the general pattern of differences among treatments remains evident, even though the overall level of responding is consequently reduced.

Although the present experiments do not reveal the basis of the observed intergroup differences in resistance to extinction, some speculation on this issue is possible. If, as Zajonc (1965) 
has suggested, the presence of another S provides a kind of "arousal," this property might be transient and influence performance only during a limited period following institution of the social conditions. Perhaps our procedures, which utilize measures obtained during a rather short period of extinction, provide an optimal situation for detection of this arousal effect. Further, an explanation of the present findings in terms of transient arousal also assumes that the effect is specific to the partner's presence in the test situation, because all pairs are maintained as cagemates.

Some further considerations of the present findings are related to their specificity to the task situation. The use of a single manipulandum for concurrent operation by two animals raises the possibility that extinction differences may depend on competition between pair members. Winslow (1944b) has reported that enhanced performance by cats in a problem box is observed when a competitor is provided. However, both Winslow (1944a), using cats, and Scott \& McCray (1967), using dogs, have reported a negative effect of competitors on speed in a runway. In opposition to explanations based on competitional dependency, the present test procedures have yielded very little of the kind of "extinction induced social interaction" reported by Davis \& Donefeld (1967). In an effort to gather more information about the effect of social interaction during test, one additional group of $10 \mathrm{Ss}$ was trained alone and, subsequently, extinguished in the presence of an untrained animal. Under procedures like those of Experiment 3, these Ss showed a mean of 131 responses to extinction, which appeared reasonably close to the 142 of alone-together Ss. Although the intent was to provide a nonresponding naive animal, the untrained rats tended to center their activity about the manipulandum and, thus, may not have provided an effect different from that of another trained animal undergoing extinction.

One further issue regarding situation specificity suggests a limitation upon the interpretation of the present findings. Although Ss originally trained together do not show the same high resistance to extinciion as do animals trained alone, it is not clear that this result is a distinctive product of training conditions. Rather, this difference might depend upon the pairing of some animals before extinction testing and the consequent provision of adaptation to the condition of "togetherness" in the test compartment. In order to evaluate this possibility, two groups of eight rats (four pairs per condition) received three 1-h sessions of adaptation together in the test compartment (with no food delivery) prior to institution of training conditions like those of Experiment 1. Under these conditions animals trained alone and extinguished together did not differ in terms of resistance to extinction from Ss both trained and extinguished together. Two similar groups received adaptation alone before training and subsequent extinction, and these groups also failed to differ significantly on extinction measures. Of course, this latter aspect of the results was disappointing in relation to our prior findings and their interpretations, but it may be noted that differences were in the predicted direction (mean of 171 responses for alone-together and 128 responses for together-together Ss), the $\mathrm{N}$ was small (8), and variability was great. Despite the failure to replicate in this extension of the original experiments, it was suggested that exposure to social pairing before administration of training conditions yielded an adaptation effect similar to that assumed to occur when Ss were actually trained together.

Although the evidence is certainly indirect, initial social pairing at the time of testing seems to yield a kind of arousal, which enhances the amount of responding a rat will display in an extinction test, and this influence may be characterized as distinctively "social." However, mere provision of a partnex at the time of extinction does not necessarily produce a response facilitation, since this effect appears dependent upon the social experience provided both prior to and during extinction.

DAVIS, $H$, \& DONEFELD, I Extinction induced social interaction in rats. Psychonomic Science, 1967, 7, 85-86.

HALL, J. F. Psychology of motivation. Chicago: Lippincott, 1961.

HOREL, J. A. TREICHLER, F. R., \& MEYER, D. $R$. Coercive behavior in the rhesus monkey. Journal of Comparative \&" Physiological Psychology, 1963, 56, 208-210.

SCOTT, J. P., \& MCCRAY, C. Allelomimetic behavior in dogs: Negative effects of competition on social facilitation. Journal of Comparative \& Physiological Psychology, 1967, 63, 316-319.

WINSLOW, C. N. The social behavior of cats, I. Competitive and aggressive behavior in an experimental runway situation. Journal of Comparative Psychology, 1944a, 37, 297-313.

WINSLOW, $C$. $N$. The social behavior of cats, II. Competitive, aggressive, and food-sharing behavior when both competitors have access to the goal. Journal of Comparative Psychology, 1944b, 37, 315-326.

ZAJONC, R. B. Social facilitation. Science, $1965,149,269-274$.

ZAJONC, R. B., \& SALES, S. M. Social facilitation of dominant and subordinate responses. Journal of Experimental Social Psychology, 1966, 2, 160-168.

\title{
Mediation of rat-mouse interspecific aggression by cage odor*
}

\author{
HARRY H. AVIS and JERRY T. TREADWAY \\ Research Laboratories, Edgewood Arsenal, Md. 21010
}

Killer rats were placed in each of four cage conditions; these were: home cage, rat-soiled cage, neutral cage, mouse-soiled cage. Latencies to attack and kill were measured, and it was found that the mouse-soiled condition was most effective in increasing latency to kill and reducing the number of killing responses.

Tollman \& King (1956) have suggested that there is an olfactory releaser involved in intraspecific aggression in mice. Ropartz (1968) has demonstrated that altering the scent of one of a pair of mice increases the latency to attack threefold and that removal of the olfactory bulb eliminates fighting altogether. Similarly, Archer (1968) has shown that putting mice into a cage recently occupied by other mice decreases the latency to attack. Thus, there appears

*In conducting the research described in this report, the investigators adhered to the "Guide for Laboratory Animal Facilities and Care," as promulgated by the Committee on the Guide for Laboratory Animal Resources, National Academy of Sciences, National Research Council. to be substantial evidence that the odor of one mouse acts to release aggressive behavior in another mouse.

In rat-mouse interspecific aggression, however, there is ample evidence that olfactory cues inhibit mousekilling. Karli, Vergnes, \& Didiergeorges (1969), in a recent review, have suggested that olfactory cues activate a system which inhibits the release of aggression. They cite evidence that rats that did not previously kill mice would do so after removal of the olfactory bulbs. Thus it appears that olfactory cues have opposite effects on these two forms of aggression.

If the above statement is accurate and if the odor of other mice (or of a neutral cage) releases aggression in the 